\title{
PARAMETER IDENTIFICATION - A NEW WAY OF ASSESSING ERRORS IN STELLAR ATMOSPHERE ANALYSIS
}

\author{
R. WEHRSE AND PH. ROSENAU \\ Institut f. Theoretische Astrophysik, \\ Tiergartenstraße 15, D 69121 Heidelberg \\ Interdisziplinäres Zentrum f. Wissenschaftl. Rechnen, \\ Im Neuenheimer Feld 368, D 69120 Heidelberg
}

\begin{abstract}
For the consistent and objective determination of stellar parameters and corresponding errors a constrained minimization approach is described which allows to take additional non-spectroscopic information into account and assures that the atmospheric structure equations are always fulfilled.
\end{abstract}

\section{Introduction}

Parameter identification and estimation is a new and fast growing field in numerical mathematics. Its results are applied in the modelling and optimization of complex systems. It has been applied very successfully e.g. in biology, robotics and chemical kinetics.

We want to adapt this approach to the analysis of stellar spectra since error estimates for stellar parameters are urgently needed e.g. for the interpretation of HR and color-color diagrams or for the assessment of metallicityage relations and of metallicity gradients in the Galaxy. Unfortunately, up to now error estimates for the effective temperatures, the gravities and in particular the element abundances appear to be quite inaccurate and inconsistent (and in many cases even rather subjective) since the various quite intricate dependencies of the emergent flux on the basic parameters have not been fully taken into account (cf. Wehrse, 1990). 


\section{Available information and assumptions}

From observations we obtain the flux $F^{o b s}\left(\lambda_{i}\right)$ at $n$ wavelengths $\lambda_{i}$ and the variances $\sigma\left(\lambda_{i}\right)$. We assume that the errors in the fluxes are normally distributed and statistically independent. In many cases, there is the additional information (e.g. from spectral classification, interferometry, or astrometry) that the gravity has to be within the range

$$
g^{l} \leq g \leq g^{u}
$$

and that the effective temperature is bracketed

$$
T_{e f f}^{l} \leq T_{e f f} \leq T_{e f f}^{u} .
$$

In essentially all cases it is required that the abundances obey the inequalities

$$
0 \leq \epsilon_{m} \leq \epsilon_{H e} \leq \epsilon_{H}, \epsilon_{m}^{l} \leq \epsilon_{m} \leq \epsilon_{m}^{u} .
$$

On the other hand, one has to assume a model which can be considered to be true. For illustration, we discuss here the most simple case and assume that the star for which the spectral analysis is carried out can be described as a spherical gas mass in hydrostatic equilibrium and that the stellar atmosphere is the geometrically thin outer layer in radiative and local thermodynamical equilibrium so that it can fully be described by the parameter vector $\mathcal{P}=\left(T_{e f f}, g, \epsilon_{i}, \ldots\right)$.

The resulting set of equations is given by the radiative transfer equation

$$
\mu \frac{d}{d z} I(\lambda, z, \mu, \mathcal{P})=-\chi(\lambda, z, \mu, \mathcal{P})(I(\lambda, z, \mu, \mathcal{P})-S(\lambda, z, \mathcal{P})),
$$

the definition of the model flux

$$
F^{\text {mod }}(\lambda, z, \mathcal{P})=2 \int_{-1}^{1} \mu I(\lambda, z, \mu, \mathcal{P}) d \mu
$$

the energy equation

$$
\frac{d}{d z} \int_{0}^{\infty} F^{\bmod }(\lambda, z, \mathcal{P}) d \lambda=0
$$

the hydrostatic equation

$$
\frac{d}{d z} P(z, \mathcal{P})=-g \rho(z, \mathcal{P})
$$

and the equation of state as well as expressions for the absorption and scattering coefficients. 


\section{Parameter and error estimation}

We consider a parameter vector $\mathcal{P}^{0}$ to contain the parameters of the star if it minimizes the objective function

$$
Z(\mathcal{P})=\sum_{i=1}^{n}\left(\frac{F^{o b s}\left(\lambda_{i}\right)-F^{\text {mod }}\left(\lambda_{i}, \mathcal{P}\right)}{\sigma\left(\lambda_{i}\right)}\right)^{2}
$$

and if simultaneously the expressions (1) - (7) are fulfilled as constraints; i.e. we require the best possible agreement between the observed and calculated spectra, the exact fulfilment of the equations for the structure of the atmosphere and the spectrum formation, and the obedience of the parameters to the limits provided by the non-spectroscopic information. In mathematical terms, the parameters are obtained by a minimization constrained by a boundary value problem.

The accuracy of the resulting parameters can now be estimated from the error ellipsoid that contains the true solution with a probability $1-\alpha$ (e.g. $95 \%$ ). It can be shown that for our set of assumptions the ellipsoid is approximated by the point set

$$
G_{L}(\alpha)=\left\{\mathcal{P}^{0}+\delta \mathcal{P} \mid J_{c} \delta \mathcal{P}=0,\left\|J_{1} \delta \mathcal{P}\right\|_{2}^{2} \leq \gamma(\alpha)\right\}
$$

with $J_{c}$ and $J_{1}$ being the Jacobi matrices of the constraints and the objective function, resp., and $\gamma(\alpha)$ being essentially the quantile of the Fisher distribution. $\|\ldots\|_{2}^{2}$ denotes the square of the Euclidean norm.

The basic idea for the approach described here dates back to C.F. Gauss. Although minimization problems with algebraic equations as constraints have already been solved by J.L. Lagrange, it was only learnt more than a century later how to treat inequalities (Karush, 1939; Kuhn and Tucker, 1951). Bock (1981) proposed an algorithm for the boundary value problem constrained case where the solution of the boundary value problem and the minimization are performed simultaneously. Our approach is based on that of Bock and involves a multiple shooting discretisation of the boundary value problems (e.g. for eqs. (4) and (7)). The resulting (in-)equality constrained minimization problem is then solved by a generalized GaussNewton iteration with line search, where the Jacobi matrices of the constraints are computed by internal numerical differentiation (Bock, 1985).

\section{Discussion}

It is evident that our approach involves the problems that it is hardly possible to estimate the errors resulting from uncertainties in the model and that it is not clear to what extent the assumptions on the flux errors 
are justified (empirical checks seem to be difficult). These problems are in fact inherent in all approaches for parameter determinations. In addition, compared to unconstrained minimization schemes (cf. Press et al., 1992) our method is quite complex and therefore the implementation is rather costly (but it has to be done only once). We think, however, that the problems are by far outweighed by the advantages: (i) the numerics is highly efficient. It is expected that - independent of the number of free parameters $n$ - typically 10 to 20 iterations are needed to achieve a relative accuracy of $\approx 10^{-3}$; a corresponding determination on the basis of a model grid and unconstrained minimization would in most cases require the calculation of $10^{n}$ atmosphere models; (ii) more importantly, the parameter determination is objective, i.e. non-spectroscopic information and empirical knowledge can be introduced consciously but are not creeping in implicitly (and perhaps unintentionally); (iii) a fully consistent error ellipsoid is obtained without much additional cost; (iv) parameters are indicated that cannot or can hardly be determined simultaneously; (v) since all Jacobi matrices are available this approach can provide help for future observations as e.g. it can show why some colors are good indicators of a parameter and others are not; (vi) although the algorithm is not guaranteed to converge to the global minimum, experience has shown that in most cases it does so. The importance of this aspect can be seen e.g. from the discussion of the nature of the carbon star G7761, cf. Gass et al. (1988); (vii) the algorithm is flexible, e.g. one of the first applications will be the empirical determination of the temperature structure of M dwarfs from ISO spectra; and (viii) last but not least the approach has a sound mathematical basis.

At the time of writing the project is still in progress but it is expected that numbers for actual stars will be available in near future.

\section{Acknowledgements}

This work has been supported by the Deutsche Agentur f. Raumfahrtangelegenheiten DARA (project 50 OR 96117).

\section{References}

Bock, H.-G. 1981, in: Modelling of Chemical Reaction Systems (Ebert, Deuflhard, Jäger, eds.), Springer Series Chemical Physics 18, 102

Bock, H.-G. 1985, PhD. thesis, Bonn University

Gass, H., Liebert, J., Wehrse, R. 1988, AA 189, 194

Karush, W. 1939, Master's Thesis, Department of Mathematics, University of Chicago

Kuhn, H. W., Tucker, A. W. 1951, in: Proc. of the Second Berkeley Symposion on Mathematical Statistics and Probability, J. Neyman (ed.), University of California Press

Press, W.H., et al. 1992, Numerical Recipes, Cambridge Univ. Press

Wehrse, R. (ed.) 1990, Accuracy of Element Abundances from Stellar Atmospheres, Lecture Notes in Physics 359, Springer Verlag 